\title{
The Design of the Infrared Spectrally Selective Low Emissivity Coating Liang PENG ${ }^{1, a}$, Jun-Sheng LI ${ }^{2, b}$, Wen-Wei ZHENG ${ }^{3, c}$ \\ ${ }^{1,2,3}$ Science and Technology on Advanced Ceramic Fibers and Composites Laboratory, National University of Defense Technology, Changsha, Hunan 410073, People's Republic of China. \\ acspl1006@163.com, ${ }^{\mathrm{b}}$ charlesljs@163.com, 'ww2247@tom.com
}

\begin{abstract}
Keywords: Infrared Stealth, Heat Radiation, Selective Low Emissivity, One-dimensional Photonic Crystal.
\end{abstract}

\begin{abstract}
The traditional low infrared emissivity materials do not have the infrared spectrally selective low emissivity characteristic, it brings the problem of compatibility of the infrared stealth and heat radiation. With the help of the one-dimensional photonic crystal(1DPC), selecting the infrared window material $\mathrm{Ge}$ and $\mathrm{MgF}_{2}$ to compose the 1DPC, and designing the infrared stealthy coating based on the structure of 1DPC. The results indicates that the wavelength-selective emission manipulated can be realized by constructing the one-dimensional heterostructure photonic crystal(1DHPC), the spectral emissivity in the atmospheric windows of 3.0 5.0 $\mu \mathrm{m}$ and $8.0 \sim 14.0 \mu \mathrm{m}$ can be as low as 0.10 and 0.08 respectively, and the spectral emissivity in the non- atmospheric windows of 5.0 8.0 $\mu \mathrm{m}$ can be as high as 0.87 . This kind of infrared stealthy coating with infrared spectrally selective low emissivity is promising for unifying the infrared stealth and radiation, and is to better realize the infrared stealth.
\end{abstract}

\section{Introduction}

As the development of the infrared detections in the modern warfare, the application of stealth technology is more and more widely. It may hinge the success or failure of the war. Among the various kinds of stealth technology, the infrared stealth is one of the important measure. The concept of the infrared stealth is to eliminate or reduce the difference of the radiation characteristics

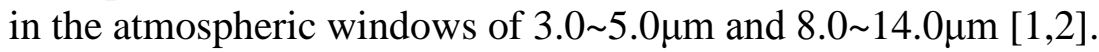

According to the Stefan-Boltzman's equation [3]:

$$
\mathrm{M}=\varepsilon \sigma \mathrm{T}^{4}
$$

There are two different approaches to reduce the intensity of the infrared radiation and realize the infrared stealth theoretically: reducing the emissivity or the temperature of the military target. Nowadays, the traditional low infrared emissivity coatings, which have the low infrared emissivity in both the atmospheric windows and non-atmospheric windows have been extensively applied [4,5]. This kind of materials certainly meet the requirements of infrared stealth, however the low infrared emissivity in the whole infrared band may influence the efficiency of heat radiation, the immediate effect is the rise of temperature. According to Eq. 1, the rise of the temperature may also lead to the increase of the infrared radiation intensity and enhance the detectability of the targets. For the traditional low infrared emissivity coatings, it is hard to unify the infrared stealth and the heat radiation. Therefore, it is important to develop infrared spectrally selective low emissivity materials, which have low emissivity in the atmospheric windows of 3.0 5.0 $\mu \mathrm{m}$ and $8.0 \sim 14.0 \mu \mathrm{m}$ to achieve infrared stealth and high emissivity in the non-atmospheric window of $5.0 \sim 8.0 \mu \mathrm{m}$ to achieve the heat radiation [6-8].

One-dimensional photonic crystal (1DPC) is made up of two kinds of materials with different refractive indices. The essential characteristic of the 1DPC is the existence of the photon forbidden band. The concept of one-dimensional heterostructure photonic crystal (1DHPC) is composed of two different kinds of 1DPCs. They can show two strong reflection peaks in two different wavelength ranges. These characteristics provides us a new method to design infrared spectrally 
selective low emissivity materials. Therefore, 1DHPC is promising for unifying the infrared stealth and heat radiation.

\section{The Stealth Principle of Photonic Crystals}

The essential characteristic of the 1DPC is the existence of the photon forbidden band as above stated. If the frequency of electromagnetic waves are located within the photon forbidden band, the electromagnetic waves cannot spread in the 1DPC. That means the photonic band gap not only can restrain the spontaneous radiation of the forbidden band frequency, but also can enhance the spontaneous radiation of the pass band. The high-reflection photon forbidden band can be removed by adjusting the period size of the 1DPC, and the forbidden band can broaden by constructing the 1DHPC. It is exactly based on this characteristic that the 1DPC can be selected as the infrared spectrally selective low emissivity material.

\section{Structure Design}

With the aid of the structure of 1DPC, this passage designed the infrared stealth coating. The structure of the coating is shown in Fig. 1, the 1DPC is the critical factor to realize the infrared spectrally selective low emissivity. We selected the $\mathrm{Si}$ as the substrate in this passage.

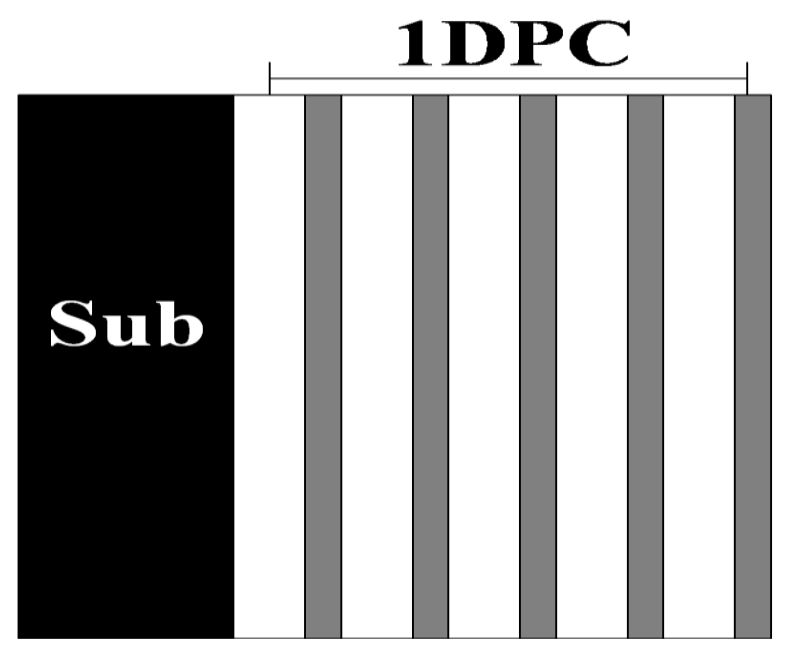

Fig. 1 Microstructure model of the coating.

The requirement of the 1DPC with infrared spectrally selective low emissivity is: low emissivity

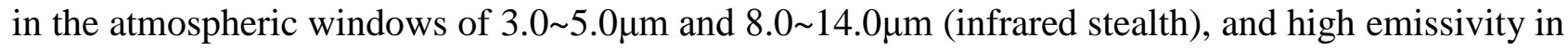
the non-atmospheric windows of 5.0 8.0 $\mu \mathrm{m}$ (heat radiation). The current design is targeted only in

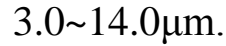

The $1 \mathrm{DPC}$ is composed of the high and low refractive index materials. In this passage, $\mathrm{Ge} / \mathrm{MgF}_{2}$ was prepared to make up the 1DPC, Ge was selected as a high refractive index material, whose refractive index is $4.02 ; \mathrm{MgF}_{2}$ was selected as a low refractive index material, whose refractive index is 1.35 .

It would be specially mentioned that, according to the Kirchoff's law [9], the relationship between the transmittance(T), reflectivity $(\mathrm{R})$ and the infrared emissivity $(\varepsilon)$ can be expressed as:

$$
\mathrm{T}+\mathrm{R}+\varepsilon=1
$$

In this passage, because the 1DPC consists of several layers, the transmittance can be seen as zero. That means the infrared emissivity can be expressed as: $\varepsilon \approx 1-\mathrm{R}$. 


\section{Calculation Model}

The reflection speciality is studied with the help of the characteristic matrix method. According to the theory of thin-film calculation, for the Jth level of the medium, the characteristic matrix is:

$$
M_{j}=\left[\begin{array}{cc}
\cos \delta_{j} & -\frac{i}{p_{j}} \sin \delta_{j} \\
-i p_{j} \sin \delta_{j} & \cos \delta_{j}
\end{array}\right]
$$

In the Eq. $3, \delta_{j}=k_{0} n_{j} d_{j} \cos \theta_{j} ; p_{j}=\sqrt{\frac{\varepsilon_{j}}{\mu_{j}}} \cos \theta_{j} ; n_{j} d_{j}$ stands for the optical thickness; $k_{0}=2 \pi / \lambda_{0}$ stands for the wave number of incident light in vacuum; $\lambda_{0}$ means the wavelength of the incident light; $\theta_{\mathrm{j}}$ stands for the angle of the light and the interface normal direction. For the 1DPC with the periods of $\mathrm{N}$, its characteristic matrix can be expressed as:

$$
\mathrm{M}=\sum_{\mathrm{j}=1}^{\mathrm{N}} \mathrm{M}_{\mathrm{j}}=\left[\begin{array}{ll}
\mathrm{M}_{11} & \mathrm{M}_{12} \\
\mathrm{M}_{21} & \mathrm{M}_{22}
\end{array}\right]
$$

The reflection coefficient of the $1 \mathrm{DPC}$ can be expressed as:

$$
r=\frac{m_{11} p_{1}+m_{12} p_{1} p_{L}-m_{21}-m_{22} P_{L}}{m_{11} p_{1}+m_{12} p_{1} p_{L}+m_{21}+m_{22} P_{L}}
$$

In Eq. 5, $\mathrm{p}_{1}=\sqrt{\frac{\varepsilon_{1}}{\mu_{1}}} \cos \theta_{1} ; \mathrm{p}_{\mathrm{L}}=\sqrt{\frac{\varepsilon_{\mathrm{L}}}{\mu_{\mathrm{L}}}} \cos \theta_{\mathrm{L}} ; \varepsilon_{1}, \mu_{1}, \varepsilon_{\mathrm{L}}, \mu_{\mathrm{L}}$ represent the dielectric constant and magnetic conductivity of the 1DPC's left and right borders respectively; $\theta_{1} \theta_{\mathrm{L}}$ represent the angle between the direction of the 1DPC's incident and transmitted wave and the normal direction of the surface.

The reflectivity can be expressed as:

$$
\mathrm{R}=|\mathrm{r}|^{2}
$$

\section{Results and Discussion}

Firstly, for the 3.0 5.0 $\mu \mathrm{m}$ band, we designed the 1DPC(A) with the central wavelength $\lambda_{0}$ is $4.0 \mu \mathrm{m}$, and the optical thickness of each layer is $\lambda_{0} / 4$. The structure of $1 \mathrm{DPC}(\mathrm{A})$ can be expressed as $\mathrm{Sub}\left|(\mathrm{HL})^{\wedge}\right|$ Air, which $\mathrm{H}$ and $\mathrm{L}$ stand for the high refractive index material $\mathrm{Ge}$ and low refractive index material $\mathrm{MgF}_{2}$ (the same below). And the curve of spectral reflectivity is shown in Fig. 2.

After that, we designed the $1 \mathrm{DPC}(\mathrm{B})$ with the central wavelength $\lambda_{0}$ is $11.0 \mu \mathrm{m}$. and the optical thickness of each layer is also $\lambda_{0} / 4$. The structure of $1 \mathrm{DPC}(\mathrm{B})$ was the same with $1 \mathrm{DPC}(\mathrm{A})$. And the curve of spectral reflectivity is shown in Fig. 3.

From Fig. 2 and Fig. 3 we can see that the high reflection region do not cover both of the atmospheric windows $(3.0 \sim 5.0 \mu \mathrm{m}$ and $8.0 \sim 14.0 \mu \mathrm{m})$, it do not meet the requirement of our design obviously. The one-dimensional heterostructure photonic crystal (1DHPC) can help us to solve the problem. we stacked up the $1 \mathrm{DPC}(\mathrm{A})$ and $1 \mathrm{DPC}(\mathrm{B})$, the structure can be expressed as $\mathrm{Sub}\left|(\mathrm{HL})^{\wedge}(\mathrm{HL})^{\wedge}\right|$ Air, and the curve of spectral reflectivity is shown in Fig. 4. We can clearly see

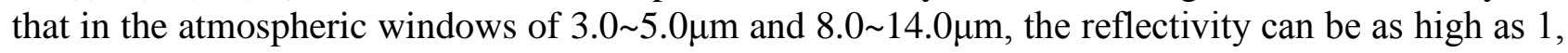
but in the non-atmospheric windows of $5.0 \sim 8.0 \mu \mathrm{m}$, the average reflectivity was also as high as 0.65 , this result may lead to the inefficiency of heat radiation. 


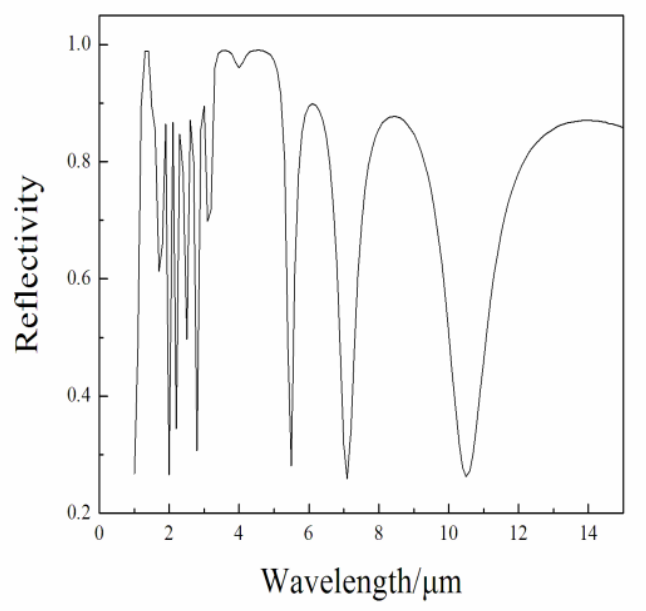

Fig. 2 The Spectral reflectivity of the 1DPC(A)

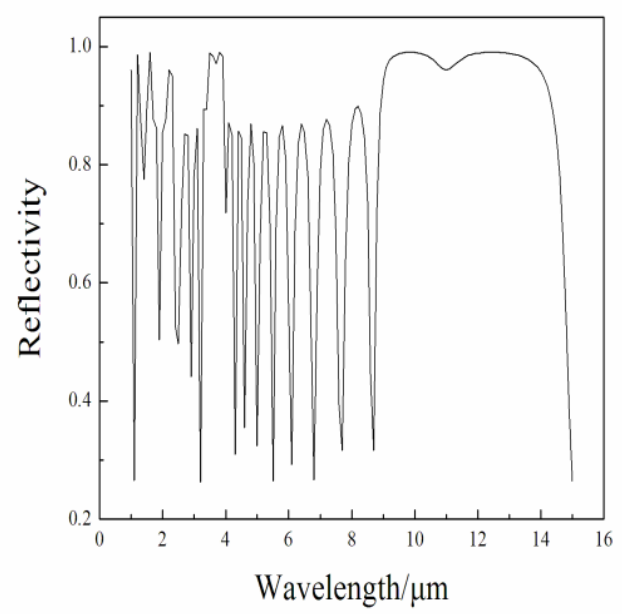

Fig. 3 The Spectral reflectivity of the $1 \mathrm{DPC}(\mathrm{B})$

Characteristic matrix is the mainly method to design the optical film [10].Considering the complexity of conducting film design and the better effect of the selective low emissivity, the method of computer simulation was adopted in the optimization design by TFCalc in this passage. The initial structure of 1DHPC (Sub|(HL) ${ }^{\wedge}(\mathrm{HL})^{\wedge} \mid$ Air) was imported into the TFCalc, the design of the 1DHPC were improved with the thickness as a variable. After the analyses and design, the structure of the 1DHPC is:

Sub|0.9H0.15L0.18H0.15L0.38H0.73L0.54H0.34L|Air, the thickness of each layer is shown in Table 1, and the curve of spectral reflectivity is shown in Fig. 5:

Tab. 1 The single layer's thickness of the 1DHPC as-designed by TFCalc

\begin{tabular}{|c|c|c|c|c|c|c|c|c|}
\hline Layer & 1 & 2 & 3 & 4 & 5 & 6 & 7 & 8 \\
\hline Material & $\mathrm{Ge}$ & $\mathrm{MgF}_{2}$ & $\mathrm{Ge}$ & $\mathrm{MgF}_{2}$ & $\mathrm{Ge}$ & $\mathrm{MgF}_{2}$ & $\mathrm{Ge}$ & $\mathrm{MgF}_{2}$ \\
\hline Thickness(nm) & 485.0 & 235.0 & 95.0 & 230.0 & 210.0 & 1130.0 & 300.0 & 520.0 \\
\hline
\end{tabular}

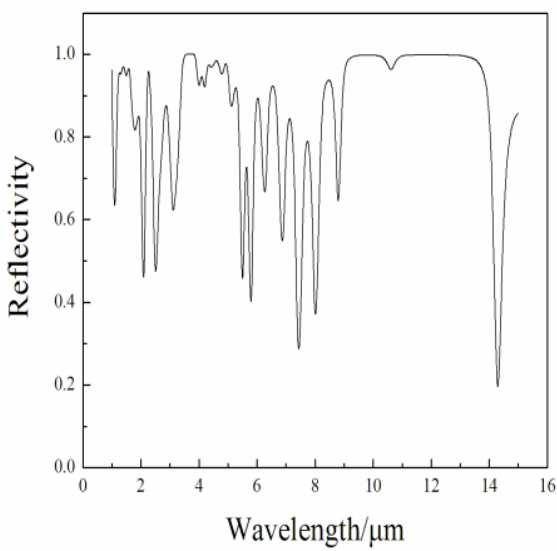

Fig. 4 The Spectral reflectivity of the 1DHPC as-designed

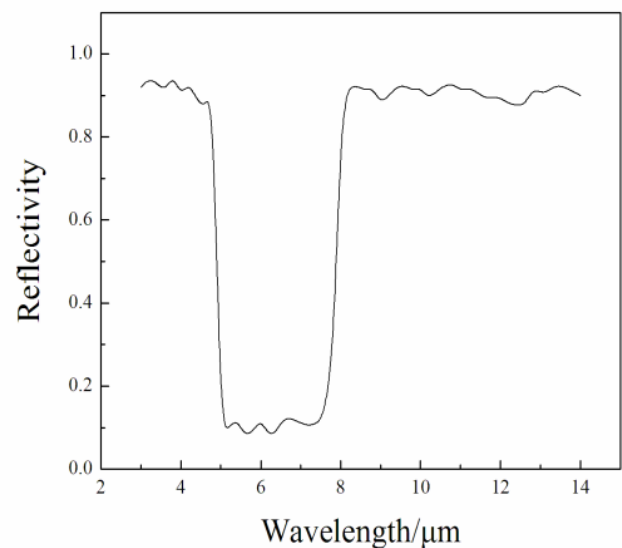

Fig. 5 The Spectral reflectivity of the1DHPC as-designed by the TFCalc 


\section{Conclusions}

In summary, $\mathrm{Ge} / \mathrm{MgF}_{2}$ was prepared to make up the one-dimensional photonic crystal(1DPC). The forbidden band was broaden by constructing the one-dimensional heterostructure photonic crystal(1DHPC), and the characteristic of infrared spectrally selective low emissivity was designed just based on the 1DHPC. The reflection spectra of the 1DPC was calculated by characteristic matrix method of thin-film optical theory and then optimized by TFCalc. The results shows that the as-prepared $\mathrm{Ge} / \mathrm{MgF}_{2}$ one-dimensional heterostructure photonic crystal has high reflectivity (low infrared emissivity) in the atmospheric windows of $3.0 \sim 5.0 \mu \mathrm{m}$ and $8.0 \sim 14.0 \mu \mathrm{m}$, and low reflectivity (high infrared emissivity) in the non-atmospheric window of 5.0 8.0 $\mu \mathrm{m}$. Therefore, the $\mathrm{Ge} / \mathrm{MgF}_{2}$ one-dimensional heterostructure photonic crystal can achieve the target of infrared stealth and heat radiation simultaneous by the atmospheric windows and non-atmospheric window. It provides a new and better way to the realization of the infrared stealth.

\section{References}

[1] X.G. Diao, W.CH. Hao, T.M. Wang, Z. Wu, J. Huang. Infrared stealth properties of low emissivity thin films, J. Aerospace Materials\&Technology. 5 (2007) 39-42.

[2] W.D. Mu, H.F. Cheng, G.P. Tang, Zh.H. Chen. The present situation and the development of thermal infrared stealth camouflage technology and materials, J. Mater Rev. 21 (2007) 114-117.

[3] M. Zhang, X.J. Yang, ,M.Y. Liu. Theoretical study on reflectivity of 1D photonic crystals of infrared stealthy materials, J. Journal of Academy of Armored Force Engineering. 5 (2009) 89-91.

[4] D.Q. Liu, H.F. Cheng, W.W. Zheng, ZH.Y. Zhang. Application of VO2 in adaptive infrared stealth technology, J. Laser\&Infrared. 11 (2012) 154-157.

[5] B.L. Liu, J.M. Shi, D.P. Zhao, W. Zhang, B. Xu. Analysis on the camouflage application of photonic crystals, J. Laser\&Infrared. 1 (2009) 42-45.

[6] H. Hogstrom, G. Forssell, G. Ribbing. Realization of selective low emittance in both thermal atmospheric windows, J. Optical Engineering. 44 (2005) 026001.

[7] L. David, C. Chan, M. Soljačić, J.D. Joannopoulos. Thermal emission and design in onedimensional periodic metallic photonic crystal slabs, J. PHYSICAL REVIEW E. 74 (2006) 016609.

[8] G.W. Zhang, G.Y. Xu, CH.J. Zhang, H.H. Wang, L.H. Hou. Infrared spectrally selective low emissivity from Ge/ZnS one-dimensional heterostructure photonic crystal, J. Optical Materials. 37 (2014) 343-346.

[9] S. Enoch, J.J. Simon, L. Escoubas. Simple layer-by-layer photonic crystal for the control of thermal emission, J. Appl. Phys. Lett. 86 (2005) 261101.

[10] M.J. Ni, N.Q. Zhao, J.X. Zhao. Transparent and high infrared reflection film having sandwich structure of $\mathrm{SiO} 2 / \mathrm{Al}: \mathrm{ZnO} / \mathrm{SiO} 2$, J. Progress in Organic Coatings. 64 (2009) 317-321. 